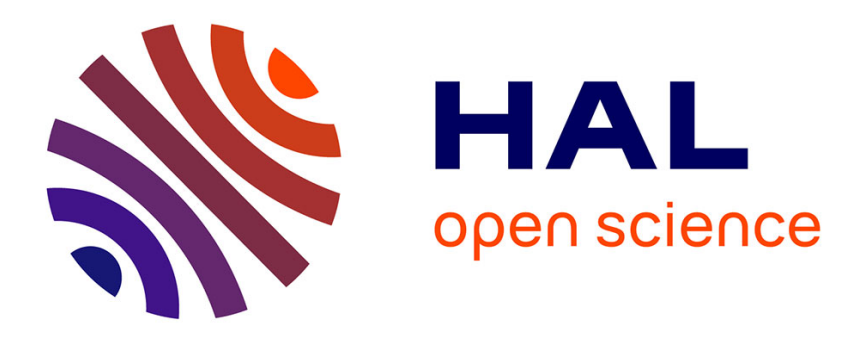

\title{
On a second conjecture of Stolarsky: the sum of digits of polynomial values
}

Manfred Madritsch, Thomas Stoll

\section{To cite this version:}

Manfred Madritsch, Thomas Stoll. On a second conjecture of Stolarsky: the sum of digits of polynomial values. Archiv der Mathematik, 2013, 102 (1), pp.49-57. 10.1007/s00013-013-0587-z . hal00959809

\section{HAL Id: hal-00959809 \\ https://hal.science/hal-00959809}

Submitted on 17 Mar 2014

HAL is a multi-disciplinary open access archive for the deposit and dissemination of scientific research documents, whether they are published or not. The documents may come from teaching and research institutions in France or abroad, or from public or private research centers.
L'archive ouverte pluridisciplinaire HAL, est destinée au dépôt et à la diffusion de documents scientifiques de niveau recherche, publiés ou non, émanant des établissements d'enseignement et de recherche français ou étrangers, des laboratoires publics ou privés. 


\title{
ON A SECOND CONJECTURE OF STOLARSKY: THE SUM OF DIGITS OF POLYNOMIAL VALUES
}

\author{
MANFRED G. MADRITSCH AND THOMAS STOLL
}

Abstract. Let $q, r \geq 2$ be integers and denote by $s_{q}$ the sum-of-digits function in base $q$. In 1978, K. B. Stolarsky conjectured that

$$
\lim _{N \rightarrow \infty} \frac{1}{N} \sum_{n \leq N} \frac{s_{2}\left(n^{r}\right)}{s_{2}(n)} \leq r .
$$

In this paper we prove this conjecture. We show that for polynomials $P_{1}(X), P_{2}(X) \in \mathbb{Z}[X]$ of degrees $r_{1}, r_{2} \geq 1$ and integers $q_{1}, q_{2} \geq 2$ we have

$$
\lim _{N \rightarrow \infty} \frac{1}{N} \sum_{n \leq N} \frac{s_{q_{1}}\left(P_{1}(n)\right)}{s_{q_{2}}\left(P_{2}(n)\right)}=\frac{r_{1}\left(q_{1}-1\right) \log q_{2}}{r_{2}\left(q_{2}-1\right) \log q_{1}} .
$$

We also present a variant of the problem to polynomial values of prime numbers.

\section{Introduction AND STATEMENT OF RESUlts}

Let $q \geq 2$ be an integer. Then every positive integer $n$ has a unique $q$-adic representation of the form

$$
n=\sum_{k=0}^{\ell} n_{k} q^{k} \quad \text { with } \quad n_{\ell} \neq 0 .
$$

We call a function $f$ a $q$-additive function if it acts only on the digits of this expansion, i.e.,

$$
f\left(\sum_{k=0}^{\ell} n_{k} q^{k}\right)=\sum_{k=0}^{\ell} f\left(n_{k} q^{k}\right) .
$$

Moreover, if this action is independent of the position of the digit, i.e., $f\left(a q^{k}\right)=f\left(a q^{j}\right)$ for $k, j \geq 0$ and $a \in\{0,1, \ldots, q-1\}$, then we call $f$ strictly $q$-additive. The most famous example of a strictly $q$-additive function is the sum-of-digits function $s_{q}$ defined by

$$
s_{q}\left(\sum_{k=0}^{\ell} n_{k} q^{k}\right)=\sum_{k=0}^{\ell} n_{k} .
$$

Date: March 17, 2014.

1991 Mathematics Subject Classification. Primary 11A63; Secondary 11N37.

Key words and phrases. sum-of-digits, mean value, polynomials, prime numbers.

The second author was supported by the Agence Nationale de la Recherche, grant ANR-10-BLAN 0103 MUNUM. 
In 1978, K. B. Stolarsky 12 studied the distribution properties of the sequence of fractions

$$
\left(s_{2}\left(n^{r}\right) / s_{2}(n)\right)_{n \geq 1},
$$

where $r \geq 2$ denotes a fixed integer. At the end of his paper, he posed two conjectures. His first conjecture was to give a proof that for all fixed $r \geq 2$ one has

$$
\liminf _{n \rightarrow \infty} \frac{s_{2}\left(n^{r}\right)}{s_{2}(n)}=0 .
$$

Hare, Laishram and Stoll 6 recently settled this conjecture and proved, more generally, that for any polynomial $P(X) \in \mathbb{Z}[X]$ with $P(\mathbb{N}) \subset \mathbb{N}$ of degree $r \geq 2$,

$$
\liminf _{n \rightarrow \infty} \frac{s_{q}(P(n))}{s_{q}(n)}=0 .
$$

Stolarsky also showed that the sequence $s_{2}\left(n^{r}\right) / s_{2}(n)$ is unbounded as $n \rightarrow$ $\infty$ (this is also true for $\frac{s_{q}(P(n))}{s_{q}(n)}$, see $|6|$ ). In very recent work, the authors 9 show that the ratio

$$
s_{q}\left(P_{1}(n)\right) / s_{q}\left(P_{2}(n)\right)
$$

for polynomials $P_{1}(X)$ and $P_{2}(X)$ of distinct degrees lies indeed dense in $\mathbb{R}^{+}$.

In [12] Stolarsky posed a second question, namely, whether

$$
\lim _{N \rightarrow \infty} \frac{1}{N} \sum_{n \leq N} \frac{s_{2}\left(n^{r}\right)}{s_{2}(n)}
$$

exists and - if it exists - to determine its value. He conjectured that the limit (1.1) exists and that it is included in the interval ]1,h]. The purpose of this paper is to prove this conjecture. More precisely, as in Hare, Laishram and Stoll [6], we show a general version. We also present a variant to polynomial values of prime numbers. Let $p_{n}$ denote the $n$-th prime, i.e., $p_{1}=2, p_{2}=3, p_{3}=5$ etc.

Our main result is the following:

Theorem 1.1. Let $q_{1}, q_{2} \geq 2$ be integers and $P_{1}(X), P_{2}(X) \in \mathbb{C}[X]$ be polynomials of degrees $r_{1}, r_{2} \geq 1$, respectively, with $P_{1}(\mathbb{N}), P_{2}(\mathbb{N}) \subset \mathbb{N}$. Then

$$
\lim _{N \rightarrow \infty} \frac{1}{N} \sum_{n \leq N} \frac{s_{q_{1}}\left(P_{1}(n)\right)}{s_{q_{2}}\left(P_{2}(n)\right)}=\frac{q_{1}-1}{q_{2}-1} \cdot\left(\frac{\log q_{1}}{\log q_{2}}\right)^{-1} \cdot \frac{r_{1}}{r_{2}} .
$$

Moreover,

$$
\lim _{N \rightarrow \infty} \frac{1}{N} \sum_{n \leq N} \frac{s_{q_{1}}\left(P_{1}\left(p_{n}\right)\right)}{s_{q_{2}}\left(P_{2}\left(p_{n}\right)\right)}=\frac{q_{1}-1}{q_{2}-1} \cdot\left(\frac{\log q_{1}}{\log q_{2}}\right)^{-1} \cdot \frac{r_{1}}{r_{2}} .
$$


Remark 1.2. In exactly the same way one can show that

$$
\lim _{N \rightarrow \infty} \frac{1}{N} \sum_{n \leq N} \frac{s_{q_{1}}\left(P_{1}\left(p_{n}\right)\right)}{s_{q_{2}}\left(P_{2}(n)\right)}=\frac{q_{1}-1}{q_{2}-1} \cdot\left(\frac{\log q_{1}}{\log q_{2}}\right)^{-1} \cdot \frac{r_{1}}{r_{2}} .
$$

All these results easily extend to strictly $q_{1^{-}}$resp. $q_{2}$-additive functions provided that the variance and the image set of the functions satisfy some suitable conditions (see [1]). These conditions are automatically verified by the sum-of-digits function.

Furthermore we remark that by replacing the theorem of Bassily and Kátai (Theorem 2.2) by analogous results due to Drmota and Steiner [4], Madritsch [7], Madritsch and Pethő [8, Drmota and Gutenbrunner [3], and Madritsch and Thuswaldner [10], respectively, one can prove analogous results for additive functions of polynomials in numeration systems that are defined via linear recurrent sequences (such as the Zeckendorf expansion), for additive functions of polynomials in numeration systems in algebraic number fields, for additive functions of polynomials in numeration systems in the quotient ring of polynomials over $\mathbb{Z}$, for additive functions of polynomials in numeration systems in the ring of polynomials over a finite field and for additive functions of polynomials in numeration systems in function fields, respectively.

\section{Preliminaries}

For the proof, we need some notation. We denote by

$$
\mu_{q}=\frac{q-1}{2} \text { and } \sigma_{q}^{2}=\frac{q^{2}-1}{12},
$$

the mean and the variance of the values of the sum-of-digits function (see 11 or [2]). We will use the letter $p$ to refer to a prime number, and use $\pi(N)$ for the number of primes up to $N$. We write $f \ll_{\omega} g$ or $f=O_{\omega}(g)$ if there exists a constant $C$ depending at most on $\omega$ such that $f(x) \leq C g(x)$ for sufficiently large $x$. If there is no such $\omega$ then the implied constant is meant to be absolute. We write $\log _{q} x$ for the logarithm to base $q$. Finally, for $A \subset \mathbb{N}$ we denote by $d(A)$ the asymptotic density of $A$, i.e.,

$$
d(A)=\lim _{N \rightarrow \infty} \frac{A \cap[1, N]}{N} .
$$

The idea of the proof of Theorem 1.1 is to use Cesàro means (see $[5]$ ). However, we cannot apply these means directly since the summands in $(1.2)$ and (1.3) could be arbitrarily large. We will therefore divide the sequence into two parts. The first part corresponds to terms where the ratio stays close to the mean value whereas the second part is made up by terms that are far away from the mean (this will be made precise in a moment). For the first part, we use Cesàro means and the following lemma, which helps us replacing the unbounded sequence by a bounded one. 
Lemma 2.1. Let $\left(x_{n}\right)_{n \in \mathbb{N}}$ be a sequence of reals and $A \subset \mathbb{N}$ a set with asymptotic density one. If

$$
\lim _{\substack{n \rightarrow \infty \\ n \in A}} x_{n}=x<\infty
$$

then

$$
\lim _{N \rightarrow \infty} \frac{1}{N} \sum_{\substack{n \leq N \\ n \in A}} x_{n}=x
$$

Proof. We define the sequence $\left(y_{n}\right)_{n \in \mathbb{N}}$ by

$$
y_{n}= \begin{cases}x_{n} & \text { if } n \in A \\ x & \text { if } n \notin A\end{cases}
$$

Since $A$ has asymptotic density one, we have

$$
\lim _{N \rightarrow \infty} \frac{1}{N} \sum_{\substack{n \leq N \\ n \in A}} x_{n}=\lim _{N \rightarrow \infty} \frac{1}{N} \sum_{n \leq N} y_{n}-\lim _{N \rightarrow \infty} \frac{1}{N} \sum_{\substack{n \leq N \\ n \notin A}} x=x .
$$

For the second part, we make use of a minor refinement of a result of Bassily and Kátai [1]. The difference to the original result is that we only suppose $P(X) \in \mathbb{C}[\bar{X}]$ instead of $P(X) \in \mathbb{Z}[X]$.

Theorem 2.2. Let $q \geq 2$ be an integer and $P(X) \in \mathbb{C}[X]$ be a polynomial of degree $r \geq 1$ with $P(\mathbb{N}) \subset \mathbb{N}$. Then

$\frac{1}{N} \#\left\{1 \leq n \leq N: \frac{s_{q}(P(n))-\mu_{q} \log _{q}\left(N^{r}\right)}{\sigma_{q}\left(\log _{q} N^{r}\right)^{\frac{1}{2}}}<t\right\} \underset{N \rightarrow \infty}{\longrightarrow} \frac{1}{\sqrt{2 \pi}} \int_{-\infty}^{t} \exp \left(-\frac{x^{2}}{2}\right) \mathrm{d} x$

and

$\frac{1}{\pi(N)} \#\left\{1 \leq p \leq N: \frac{s_{q}(P(p))-\mu_{q} \log _{q}\left(N^{r}\right)}{\sigma_{q}\left(\log _{q} N^{r}\right)^{\frac{1}{2}}}<t\right\} \underset{N \rightarrow \infty}{\longrightarrow} \frac{1}{\sqrt{2 \pi}} \int_{-\infty}^{t} \exp \left(-\frac{x^{2}}{2}\right) \mathrm{d} x$.

Proof. To prove this refinement we proceed in two steps, starting with an application of the following

Lemma 2.3 ( [11, Satz 6.9]). Let $P(X) \in \mathbb{C}[X]$ be a polynomial of degree $r$. If $P(n) \in \mathbb{Z}$ for all $n \in \mathbb{Z}$, then there exists $c_{0}, \ldots, c_{r} \in \mathbb{N}$ such that

$$
P(x)=\sum_{i=0}^{r} c_{i}\left(\begin{array}{l}
x \\
i
\end{array}\right) .
$$

Thus, in order to have $P(\mathbb{N}) \subset \mathbb{N}$, we get that $P(X) \in \mathbb{Q}[X]$.

Secondly, we take a closer look at the proof the Lemma 5 in [1]. We use the same notation throughout the rest of the proof. Let $c \in \mathbb{N}$ be the 
smallest positive integer such that $c P(X)=\tilde{P}(X) \in \mathbb{Z}[X]$. By replacing $P$ everywhere with $\tilde{P} / c$ we reach at Equation 4.10 of [1], which then reads

$$
\sum_{n \leq x} t\left(\frac{\tilde{P}(n)}{c}\right)=\sum_{M} T_{M} \sum_{n \leq x} e\left(\frac{A_{M}}{H_{M}} \frac{\tilde{P}(n)}{c}\right)=\sum_{M} T_{M} \sum_{n \leq x} e\left(\frac{A_{M}^{\prime}}{H_{M} c^{\prime}} \tilde{P}(n)\right)
$$

and

$$
\sum_{p \leq x} t\left(\frac{\tilde{P}(p)}{c}\right)=\sum_{M} T_{M} \sum_{p \leq x} e\left(\frac{A_{M}}{H_{M}} \frac{\tilde{P}(p)}{c}\right)=\sum_{M} T_{M} \sum_{p \leq x} e\left(\frac{A_{M}^{\prime}}{H_{M} c^{\prime}} \tilde{P}(p)\right)
$$

with

$$
A_{M}^{\prime}=\frac{A_{M}}{\left(A_{M}, c\right)}, \quad c^{\prime}=\frac{c}{\left(A_{M}, c\right)} \quad \text { and } \quad\left(A_{M}^{\prime}, H_{M} c^{\prime}\right)=1 .
$$

Let $q=p_{1}^{e_{1}} \cdots p_{s}^{e_{s}}$ be the prime decomposition of $q$. If $q \nmid m_{h}$, then $p_{t}^{e_{t}} \nmid m_{h}$ for some $1 \leq t \leq s$. Thus

$$
H_{M} c^{\prime}\left(m_{h}+q^{\ell_{h}-\ell_{h-1}} m_{h-1}+\cdots+m_{1} q^{\ell_{h}-\ell_{1}}\right)=A_{M}^{\prime} q^{\ell_{h}+1} .
$$

Since $p_{t}^{e_{t}} \nmid m_{h}$ and $\left(A_{M}^{\prime}, H_{M} c^{\prime}\right)=1$ we get that $p_{t}^{\ell_{t} e_{t}} \mid H_{M} c^{\prime}$. Thus $H_{M} c^{\prime} \geq$ $q^{\eta \ell_{h}}$ with $\eta=e_{t} \log _{q} p_{t}$. Similarly we get that $H_{M} \geq q^{\eta \ell_{s}}$ holds if $q \nmid m_{s}$ and $m_{s+1}=\cdots=m_{h}=0$. Therefore we may apply Lemma 1 and 2 of [1] and the Theorem follows in the same way.

\section{Proof of Theorem 1.1}

In the sequel, assume that $N \geq 2$ is a fixed real number. For a given integer $q \geq 2$ and a given polynomial $P(X) \in \mathbb{Z}[X]$ (with $P(\mathbb{N}) \subset \mathbb{N}$ ) of degree $r \geq 1$ we define $A_{q, P}=A_{q, P}(N)$ to be the set of integers $n$ with $1 \leq n \leq N$ such that $s_{q}(P(n))$ is close to its mean value, i.e.,

$$
A_{q, P}:=\left\{1 \leq n \leq N:\left|s_{q}(P(n))-\mu_{q} r \log _{q} N\right| \leq \sigma_{q}\left(r \log _{q} N\right)^{\frac{3}{4}}\right\} .
$$

(We remark that in fact any exponent larger than $\frac{1}{2}$ in place of $\frac{3}{4}$ would have done the job.) In a similar way, we define $B_{q, P}=B_{q, P}(N)$ to be the set of integers $n$ with $1 \leq n \leq N$ such that $s_{q}\left(P\left(p_{n}\right)\right)$ is close to its mean value (note that by the prime number theorem we have $p_{N} \sim N \log N$, as $N \rightarrow \infty)$, i.e.,

$B_{q, P}:=\left\{1 \leq n \leq N:\left|s_{q}\left(P\left(p_{n}\right)\right)-\mu_{q} r \log _{q}(N \log N)\right| \leq \sigma_{q}\left(r \log _{q}(N \log N)\right)^{\frac{3}{4}}\right\}$.

In order to be able to apply the properties of the Cesàro mean we need that both the numerator and the denominator of the ratios in $(1.2)$ and $(1.3)$ are near the mean. We first show that for $N \rightarrow \infty$ we have $\# B_{q, P} \sim N$ and $\# A_{q, P} \sim N$. We then use asymptotic densities to show that there are only few elements in $[1, N] \backslash\left(A_{q_{1}, P_{1}} \cap A_{q_{2}, P_{2}}\right)$ resp. $[1, N] \backslash\left(B_{q_{1}, P_{1}} \cap B_{q_{2}, P_{2}}\right)$. We will then be able to restrict our attention to $A_{q_{1}, P_{1}} \cap A_{q_{2}, P_{2}}$ resp. $B_{q_{1}, P_{1}} \cap B_{q_{2}, P_{2}}$ in the end. 
We start with an application of Theorem 2.2. As $N \rightarrow \infty$, we get that

$$
\begin{gathered}
\#\left([1, N] \backslash A_{q, P}\right)=\#\left\{1 \leq n \leq N:\left|\frac{s_{q}(P(n))-\mu_{q} r \log _{q} N}{\sigma_{q}\left(r \log _{q} N\right)^{\frac{1}{2}}}\right|>\left(r \log _{q} N\right)^{\frac{1}{4}}\right\} \\
\ll N \int_{\left(r \log _{q} N\right)^{\frac{1}{4}}}^{\infty} \exp \left(-\frac{x^{2}}{2}\right) \mathrm{d} x .
\end{gathered}
$$

Thus the number of elements that lie not in $A_{q, P}$ can be estimated by the tail of the normal distribution. We have

$$
\int_{t}^{\infty} \exp \left(-\frac{x^{2}}{2}\right) \mathrm{d} x \leq \int_{t}^{\infty} \frac{x}{t} \exp \left(-\frac{x^{2}}{2}\right) \mathrm{d} x=\frac{\exp \left(-\frac{t^{2}}{2}\right)}{t}
$$

where we have used that $0<t \leq x$. Therefore,

$$
\begin{aligned}
\#\left([1, N] \backslash A_{q, P}\right) & \ll N \exp \left(-\frac{\left(r \log _{q} N\right)^{\frac{1}{2}}}{2}\right)\left(r \log _{q} N\right)^{-\frac{1}{4}} \\
& \ll \frac{N}{\left(r \log _{q} N\right)^{\frac{5}{4}}} \ll_{q, P} \frac{N}{(\log N)^{\frac{5}{4}}} .
\end{aligned}
$$

The same calculation also shows that

$$
\begin{aligned}
& \#\left([1, N] \backslash B_{q, P}\right) \\
& =\#\left\{1 \leq n \leq N:\left|\frac{s_{q}\left(P\left(p_{n}\right)\right)-\mu_{q} r \log _{q}(N \log N)}{\sigma_{q}\left(r \log _{q}(N \log N)\right)^{\frac{1}{2}}}\right|>\left(r \log _{q}(N \log N)\right)^{\frac{1}{4}}\right\} \\
& \quad \ll_{q, P} \frac{N}{(\log N)^{\frac{5}{4}}} .
\end{aligned}
$$

Recall the setting of Theorem 1.1. The prime number theorem (in the form $p_{N} \sim N \log N$ ) and a comparison of the lengths of the expansions give

$$
\max \left(\frac{s_{q_{1}}\left(P_{1}(n)\right)}{s_{q_{2}}\left(P_{2}(n)\right)}, \frac{s_{q_{1}}\left(P_{1}\left(p_{n}\right)\right)}{s_{q_{2}}\left(P_{2}\left(p_{n}\right)\right)}\right) \ll_{q_{1}, P_{1}} \log N, \quad N \rightarrow \infty,
$$

uniformly for all $n$ with $1 \leq n \leq N$. Hence, we get from (3.1) that

$$
\begin{aligned}
\frac{1}{N} \sum_{\substack{n \leq N \\
n \notin A_{q_{1}, P_{1}} \cap A_{q_{2}, P_{2}}}} \frac{s_{q_{1}}\left(P_{1}(n)\right)}{s_{q_{2}}\left(P_{2}(n)\right)} & \ll_{q_{1}, P_{1}} \frac{\log N}{N}\left(\sum_{\substack{n \leq N \\
n \notin A_{q_{1}, P_{1}}}} 1+\sum_{\substack{n \leq N \\
n \notin A_{q_{2}, P_{2}}}} 1\right) \\
& \ll_{q_{1}, q_{2}, P_{1}}(\log N)(\log N)^{-\frac{5}{4}}=o(1),
\end{aligned}
$$

and similarly from 3.2 that

$$
\frac{1}{N} \sum_{\substack{n \leq N \\ n \notin B_{q_{1}}, P_{1} \cap B_{q_{2}, P_{2}}}} \frac{s_{q_{1}}\left(P_{1}\left(p_{n}\right)\right)}{s_{q_{2}}\left(P_{2}\left(p_{n}\right)\right)}=o(1) .
$$


Now we turn to the elements which are in $A_{q_{1}, P_{1}} \cap A_{q_{2}, P_{2}}$. By the definitions of these sets we get for all $n \in A_{q_{1}, P_{1}} \cap A_{q_{2}, P_{2}}$ (note that the denominator is positive),

$$
\begin{aligned}
\frac{s_{q_{1}}\left(P_{1}(n)\right)}{s_{q_{2}}\left(P_{2}(n)\right)} \leq & \frac{\mu_{q_{1}} r_{1} \log _{q_{1}} N+\sigma_{q_{1}}\left(r_{1} \log _{q_{1}} N\right)^{\frac{3}{4}}}{\mu_{q_{2}} r_{2} \log _{q_{2}} N-\sigma_{q_{2}}\left(r_{2} \log _{q_{2}} N\right)^{\frac{3}{4}}} \\
& =\frac{\frac{\mu_{q_{1}} r_{1}}{\log q_{1}}+\sigma_{q_{1}} r_{1}^{\frac{3}{4}}\left(\log _{q_{1}} N\right)^{-\frac{1}{4}}\left(\log q_{1}\right)^{-\frac{3}{4}}}{\frac{\mu_{q_{2}} r_{2}}{\log q_{2}}-\sigma_{q_{2}} r_{2}^{\frac{3}{4}}\left(\log _{q_{2}} N\right)^{-\frac{1}{4}}\left(\log q_{2}\right)^{-\frac{3}{4}}}
\end{aligned}
$$

and

$$
\frac{s_{q_{1}}\left(P_{1}(n)\right)}{s_{q_{2}}\left(P_{2}(n)\right)} \geq \frac{\frac{\mu_{q_{1}} r_{1}}{\log q_{1}}-\sigma_{q_{1}} r_{1}^{\frac{3}{4}}\left(\log _{q_{1}} N\right)^{-\frac{1}{4}}\left(\log q_{1}\right)^{-\frac{3}{4}}}{\frac{\mu_{q_{2}} r_{2}}{\log q_{2}}+\sigma_{q_{2}} r_{2}^{\frac{3}{4}}\left(\log _{q_{2}} N\right)^{-\frac{1}{4}}\left(\log q_{2}\right)^{-\frac{3}{4}}} .
$$

This can be rephrased as follows. Let $\left(N_{k}\right)_{k \geq 0}$ be any sequence of reals with $\lim _{k \rightarrow \infty} N_{k}=\infty$ and let $\left(n_{k}\right)_{k \geq 0}$ be any sequence of integers with $n_{k} \in A_{q_{1}, P_{1}}\left(N_{k}\right) \cap A_{q_{2}, P_{2}}\left(N_{k}\right)$. Then

$$
\lim _{k \rightarrow \infty} \frac{s_{q_{1}}\left(P_{1}\left(n_{k}\right)\right)}{s_{q_{2}}\left(P_{2}\left(n_{k}\right)\right)}=\frac{\mu_{q_{1}} \log q_{2}}{\mu_{q_{2}} \log q_{1}} \cdot \frac{r_{1}}{r_{2}} .
$$

A similar calculation shows that

$$
\begin{aligned}
\frac{s_{q_{1}}\left(P_{1}\left(p_{n}\right)\right)}{s_{q_{2}}\left(P_{2}\left(p_{n}\right)\right)} \leq & \frac{\mu_{q_{1}} r_{1} \log _{q_{1}}(N \log N)+\sigma_{q_{1}}\left(r_{1} \log _{q_{1}}(N \log N)\right)^{\frac{3}{4}}}{\mu_{q_{2}} r_{2} \log _{q_{2}}(N \log N)-\sigma_{q_{2}}\left(r_{2} \log _{q_{2}}(N \log N)\right)^{\frac{3}{4}}} \\
& =\frac{\frac{\mu_{q_{1}} r_{1}}{\log q_{1}}\left(1+\frac{\log \log N}{\log N}\right)+\sigma_{q_{1}} r_{1}^{\frac{3}{4}}\left(\log q_{1}\right)^{-\frac{3}{4}}(\log N)^{-\frac{1}{4}}\left(1+\frac{\log \log N}{\log N}\right)^{\frac{3}{4}}}{\frac{\mu_{q_{2}} r_{2}}{\log q_{2}}\left(1+\frac{\log \log N}{\log N}\right)-\sigma_{q_{2}} r_{2}^{\frac{3}{4}}\left(\log q_{2}\right)^{-\frac{3}{4}}(\log N)^{-\frac{1}{4}}\left(1+\frac{\log \log N}{\log N}\right)^{\frac{3}{4}}} .
\end{aligned}
$$

In a similar way we get the lower bound with the signs reversed. Again, we obtain as limit

$$
\lim _{k \rightarrow \infty} \frac{s_{q_{1}}\left(P_{1}\left(p_{n_{k}}\right)\right)}{s_{q_{2}}\left(P_{2}\left(p_{n_{k}}\right)\right)}=\frac{\mu_{q_{1}} \log q_{2}}{\mu_{q_{2}} \log q_{1}} \cdot \frac{r_{1}}{r_{2}} .
$$

Now, since by (3.1) and (3.2) we have that

$$
\# A_{q, P}(N) / N \sim \# B_{q, P}(N) / N \sim 1
$$

as $N \rightarrow \infty$, the sets $\mathcal{A}_{q, P}=\bigcup_{N \geq 1} A_{q, P}(N)$ and $\mathcal{B}_{q, P}=\bigcup_{N \geq 1} B_{q, P}(N)$ satisfy $d\left(\mathcal{A}_{q, P}\right)=d\left(\mathcal{B}_{q, P}\right)=1$, and therefore

$$
d\left(\mathcal{A}_{q_{1}, P_{1}} \cap \mathcal{A}_{q_{2}, P_{2}}\right)=d\left(\mathcal{B}_{q_{1}, P_{1}} \cap \mathcal{B}_{q_{2}, P_{2}}\right)=1 .
$$

By Lemma 2.1, the limit for $n \rightarrow \infty$ is not altered when we only look at those $n$ that lie in these subsets of asymptotic density one. Thus we get for 
the Cesàro mean that

$$
\lim _{N \rightarrow \infty} \frac{1}{N} \sum_{\substack{n \leq N \\ n \in \mathcal{A}_{q_{1}}, P_{1} \cap \mathcal{A}_{q_{2}, P_{2}}}} \frac{s_{q_{1}}\left(P_{1}(n)\right)}{s_{q_{2}}\left(P_{2}(n)\right)}=\frac{\mu_{q_{1}} \log q_{2}}{\mu_{q_{2}} \log q_{1}} \cdot \frac{r_{1}}{r_{2}} .
$$

A combination of $(3.3)$ and $(3.5)$ yields

$$
\begin{aligned}
& \lim _{N \rightarrow \infty} \frac{1}{N} \sum_{n \leq N} \frac{s_{q_{1}}\left(P_{1}(n)\right)}{s_{q_{2}}\left(P_{2}(n)\right)} \\
& =\lim _{N \rightarrow \infty} \frac{1}{N} \sum_{\substack{n \leq N \\
n \in \mathcal{A}_{q_{1}, P_{1} \cap \mathcal{A}_{q_{2}, P_{2}}}}} \frac{s_{q_{1}}\left(P_{1}(n)\right)}{s_{q_{2}}\left(P_{2}(n)\right)}+\lim _{N \rightarrow \infty} \frac{1}{N} \sum_{\substack{n \leq N \\
n \notin \mathcal{A}_{q_{1}, P_{1}} \cap \mathcal{A}_{q_{2}, P_{2}}}} \frac{s_{q_{1}}\left(P_{1}(n)\right)}{s_{q_{2}}\left(P_{2}(n)\right)} \\
& =\frac{\mu_{q_{1}} \log q_{2}}{\mu_{q_{2}} \log q_{1}} \cdot \frac{r_{1}}{r_{2}}
\end{aligned}
$$

which proves $(1.2)$, and similarly we get $(1.3)$. This completes the proof of Theorem 1.1.

\section{Concluding Remarks}

The above results do not hold in general for arbitrary $q$-additive functions. For example, let $f: \mathbb{N} \rightarrow \mathbb{N}$ be such that $f(n)=1$ for $n \geq 0$. This function is $q$-additive (for any $q \geq 2$ ) as it puts 1 on the least significant digit of $n$ and 0 on all other digits. Then, for each $r \geq 1$, we have that

$$
\lim _{N \rightarrow \infty} \frac{1}{N} \sum_{n=1}^{N} \frac{f\left(n^{r}\right)}{f(n)}=1 \text {. }
$$

On the other hand, if $f: \mathbb{N} \rightarrow \mathbb{N}$ is $q$-additive with $f\left(a q^{k}\right)=2^{k}$ for $a \in$ $\{0, \ldots, q-1\}$ and $k \geq 0$ then for each $r \geq 2$,

$$
\lim _{N \rightarrow \infty} \frac{1}{N} \sum_{n=1}^{N} \frac{f\left(n^{r}\right)}{f(n)}=\infty .
$$

In order to get other non-trivial values for the limit, the values of $f$ must depend on the position $k$ as well as on the digit $a$. We conclude our discussion with the following

Conjecture. Let $q, r \geq 2$ be integers. Then for each real $\ell \in[1, \infty)$ there exists a q-additive function $f: \mathbb{N} \rightarrow \mathbb{N}$ such that

$$
\lim _{N \rightarrow \infty} \frac{1}{N} \sum_{n=1}^{N} \frac{f\left(n^{r}\right)}{f(n)}=\ell .
$$

\section{ACKNOWLEDGMENT}

The authors want to thank Peter J. Grabner (Graz University of Technology) and Kevin G. Hare (University of Waterloo) for fruitful discussions on this problem. 


\section{REFERENCES}

[1] N. L. Bassily and I. Kátai, Distribution of the values of q-additive functions on polynomial sequences, Acta Math. Hungar. 68 (1995), no. 4, 353-361.

[2] M. Drmota, The joint distribution of q-additive functions, Acta Arith. 100 (2001), no. $1,17-39$.

[3] M. Drmota and G. Gutenbrunner, The joint distribution of Q-additive functions on polynomials over finite fields, J. Théor. Nombres Bordeaux 17 (2005), no. 1, 125-150.

[4] M. Drmota and W. Steiner, The Zeckendorf expansion of polynomial sequences, J. Théor. Nombres Bordeaux 14 (2002), no. 2, 439-475.

[5] G. H. Hardy, Divergent series, Éditions Jacques Gabay, Sceaux, 1992, With a preface by J. E. Littlewood and a note by L. S. Bosanquet, Reprint of the revised (1963) edition.

[6] K. G. Hare, S. Laishram, and T. Stoll, Stolarsky's conjecture and the sum of digits of polynomial values, Proc. Amer. Math. Soc. 139 (2011), no. 1, 39-49.

[7] M. G. Madritsch, Asymptotic normality of b-additive functions on polynomial sequences in number systems, Ramanujan J. 21 (2010), no. 2, 181-210.

[8] M. G. Madritsch and A. Pethő, Asymptotic normality of additive functions on polynomial sequences in canonical number systems, J. Number Theory 131 (2011), no. 9, 1553-1574.

[9] M. G. Madritsch and T. Stoll, On simultaneous digital expansions of polynomial values, Acta Math. Hungar. (to appear).

[10] M. G. Madritsch and J. M. Thuswaldner, Additive functions for number systems in function fields, Finite Fields Appl. 16 (2010), no. 3, 204-229.

[11] A. Pethő, Algebraische Algorithmen, Friedr. Vieweg \& Sohn, Braunschweig, 1999.

[12] K. B. Stolarsky, The binary digits of a power, Proc. Amer. Math. Soc. 71 (1978), no. $1,1-5$.

1. Université de Lorraine, Institut Elie Cartan de Lorraine, UMR 7502, VAndoeuvre-Lès-Nancy, F-54506, France; 2. CNRS, Institut Elie Cartan de Lorraine, UMR 7502, VANDOEUVRE-LÈS-NANCY, F-54506, FranCE

E-mail address: manfred.madritsch@univ-lorraine.fr

1. Université de Lorraine, Institut Elie Cartan de Lorraine, UMR 7502, VANDOEUVRE-LÈS-NANCY, F-54506, France; 2. CNRS, Institut Elie Cartan de Lorraine, UMR 7502, VANDOEUVRE-LÈS-NANCY, F-54506, France

E-mail address: thomas.stoll@univ-lorraine.fr 